\title{
Population structure and dispersal patterns of loggerhead sea turtles Caretta caretta in Tunisian coastal waters, central Mediterranean
}

\author{
Olfa Chaieb ${ }^{1,2, *}$, Ali Elouaer ${ }^{2}$, Fulvio Maffucci ${ }^{3}$, Sami Karaa ${ }^{4}$, \\ Mohamed Nejmeddine Bradai ${ }^{2}$, Hedia ElHili ${ }^{2}$, Flegra Bentivegna ${ }^{3}$, Khaled Said ${ }^{1}$, \\ Noureddine Chatti ${ }^{1}$ \\ ${ }^{1}$ Laboratory of Genetics, Biodiversity and Bio-resources Development -LR11ES41-, Higher Institute of Biotechnology \\ of Monastir, University of Monastir, 5000 Tunisia \\ ${ }^{2}$ Laboratory of Marine Biodiversity and Biotechnology, National Institute of Sea Sciences and Technologies, Tunisia \\ ${ }^{3}$ Stazione Zoologica ‘Anton Dohrn', Villa Communale, Naples, Italy \\ ${ }^{4}$ Faculty of Sciences, University of Sfax, Tunisia
}

\begin{abstract}
Understanding the dispersal pattern and mechanisms affecting the mixing of Endangered loggerhead sea turtles Caretta caretta is a key issue for establishing sound conservation strategies. Loggerhead turtles are commonly encountered all along the Tunisian coast and particularly in the Gulf of Gabès, which is one of the most important foraging habitats for this species in the Mediterranean Sea. We conducted extensive sampling of 175 juvenile and adult loggerhead turtles that were stranded or incidentally captured in the areas along the northern (NTC), eastern (Gulf of Hammamet, GHAM) and southern (Gulf of Gabès, GGAB) Tunisian coast over a 6 yr period (2004 to 2009). A 380 bp fragment of the mitochondrial DNA control region was sequenced and analysed. Seven haplotypes were revealed, and a gradual variation in haplotype frequencies was observed among the studied areas. The extreme samples (NTC and GGAB) differed genetically from each other, while both were similar to the central sample (GHAM). This finding was confirmed by mixed stock analysis estimates, which suggested that the proportions of Atlantic turtles were very high in the NTC and decreased southward, while the proportions of Mediterranean contributions were higher in the GGAB and decreased in the opposite direction. The observed pattern of genetic variation and turtle distribution, which is probably related to differences in geomorphology and sea surface currents among these coastal areas, should be considered in future management plans.
\end{abstract}

KEY WORDS: Loggerhead sea turtle $\cdot$ Mitochondrial DNA $\cdot$ Foraging $\cdot$ Dispersal $\cdot$ Mixed stock analysis

\section{INTRODUCTION}

A fundamental research priority in conservation biology is to investigate the dispersal patterns of endangered organisms in order to establish sound conservation strategies that take into consideration various life-history stages, habitats and threats
(Bowen et al. 2005, Naro-Maciel et al. 2007, Carreras et al. 2011). This is especially challenging when dealing with large marine migratory animals, such as loggerhead sea turtles Caretta caretta, that have a complex life cycle. This cycle is characterized by a series of ecological transitions associated with continuous movements and habitat shifts. After hatching, young 
loggerhead turtles are thought to drift passively for several years in the ocean zones during the oceanic developmental phase (Bolten \& Balazs 1995). Then, as larger juveniles, an unknown proportion recruits to neritic habitats to complete their development in the vicinity of their natal rookeries (neritic developmental phase; Bolten 2003, Bowen et al. 2004), while others remain in the pelagic zone. This ontogenetic switch from oceanic to neritic habitat is not immutable, and juveniles may return to the oceanic province (Casale et al. 2007, Monzon-Arguello et al. 2010, Schofield et al. 2010). Upon reaching sexual maturity, adult turtles begin periodical migration from the foraging grounds to the nesting habitats, with females exhibiting natal homing behaviour (Bowen et al. 1993). This philopatric behaviour results in significant differences in mtDNA haplotype frequencies among nesting colonies (Bowen \& Karl 2007 ) that can be used to link feeding populations to their rookeries of origin (Norman et al. 1994).

The loggerhead turtle is the most abundant marine turtle species in the Mediterranean Sea (Margaritoulis et al. 2003), and is known to host 2 genetically distinct groups: one from the Mediterranean nesting areas and the other from the Atlantic rookeries (Laurent et al. 1998, Carreras et al. 2006). Previous studies based on satellite tracking, genetic analyses and tagging have shown the complexity of the mixing pattern of these 2 reproductively independent groups within the Mediterranean foraging areas. Juveniles from the Atlantic rookeries enter the Mediterranean during the oceanic developmental phase to feed in the western and eastern pelagic zones where they mix with local individuals. However, only a small proportion of Atlantic turtles recruit to the neritic foraging habitats (Maffucci et al. 2006, Casale et al. 2008b). Upon sexual maturity, they leave this basin to complete their development and to reproduce in the Atlantic Ocean (Laurent et al. 1998, Carreras et al. 2006, Maffucci et al. 2006, Casale et al. 2008b). Factors such as sea surface currents, water salinity and topography have been suggested to shape the distribution of these independent populations within the Mediterranean Sea (Bentivegna 2002, Cardona et al. 2005, Carreras et al. 2006, Revelles et al. 2007a, 2008, Hays et al. 2010).

The Tunisian coast represents a transition zone between western and eastern Mediterranean basins through the Siculo-Tunisian Strait. In this area, 2 principal water masses with different characteristics (temperature and salinity) flow in opposite directions. The waters $(0-100 \mathrm{~m}$ depth) originating from the Atlantic move eastward along the Algerian coast and flow into the eastern basin, and the Levantine Intermediate Water (300-350 m depth) formed in the eastern basin, flows westwards (Béranger et al. 2004 and references therein). Furthermore, Tunisian littoral topography is highly variable. The sea bottoms of the northern littoral zone (in the western basin) are mainly rocky with reduced continental shelf, while those of the eastern and southern coasts (in the eastern basin) are sandy to sandy-muddy and with a progressive larger continental shelf to the south (Ben Mustapha et al. 2003). These different features result in the distinction of 3 geographical sub-areas: the northern zone (NTC), the Gulf of Hammamet (GHAM) and the Gulf of Gabès (GGAB; Hattour 1999) (Fig. 1). These areas are characterized by a variety of habitats and a high biodiversity.

Loggerhead turtles are encountered all along the Tunisian coast, although the highest concentrations are found in the south, especially in the GGAB. This gulf is of economic interest for the country as its fishery production, estimated at $46569 \mathrm{t}$, represents about $69 \%$ of the Tunisian total production (Ben

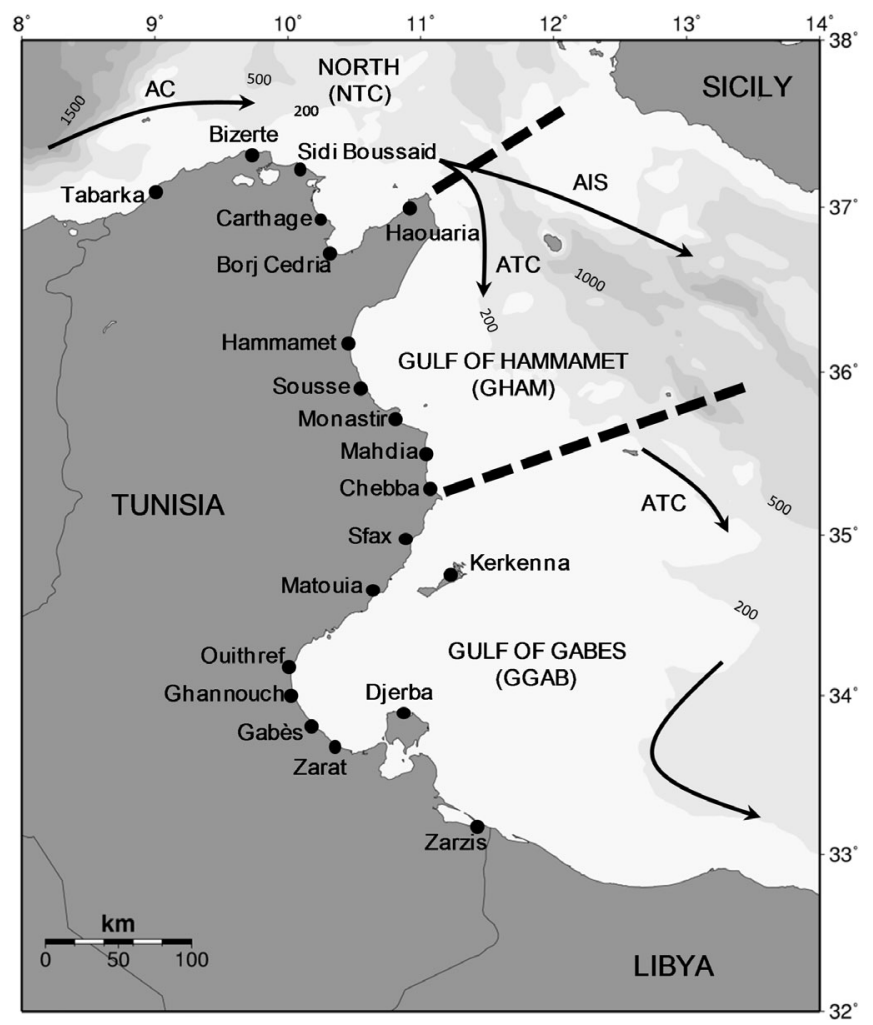

Fig. 1. Caretta caretta. Sampling sites. Bathymetry (isobaths) and sea surface currents (arrows, Béranger et al. 2004) are indicated. Black circles are sampling locations of stranded turtles, and dashed lines show boundaries of the study areas. ATC: Atlantic Tunisian Current; AC: Algerian Current; AIS: Atlantic Ionian Stream 
Salem et al. 2002). Some studies have been carried out on immature loggerhead turtles encountered in Tunisian waters but these were mostly limited to the monitoring of stranding events and to interaction with fisheries. For instance, Bradai et al. (2008) recorded 180 stranded dead loggerhead turtles on the Tunisian coast during 2 monitoring campaigns (2006 and 2007), 93\% of which were in the GGAB. In this region, heavy fisheries bycatch causes high mortality of juvenile and sub-adult turtles (Jribi et al. 2007, Echwikhi et al. 2010). Additionally, tagging and satellite tracking of loggerhead turtles have provided some insights into the spatial ecology, migratory movements and habitat selection of the individuals that utilize the Tunisian foraging grounds (Broderick et al. 2007, Hochscheid et al. 2007, Zbinden et al. 2007, 2011). However, to date, only a limited effort has been made to genetically characterize the loggerhead turtle population frequenting Tunisian habitats. In a recent work, Chaieb et al. (2010) focused only on a nesting population sampled from the main nesting site (Kuriat Islands). Casale et al. (2008b) and Laurent et al. (1998) analysed turtles frequenting eastern Tunisian foraging habitats without specifying their exact origins. Moreover, they did not extend their sampling to the western Tunisian coast, which has never been characterized. Furthermore, given the particular pattern of sea surface currents and water masses thought to play a relevant role in the dispersal of immature loggerhead sea turtles (Davenport \& Clough 1986, Bolten 2003) in the Siculo-Tunisian Strait area connecting eastern and western Mediterranean, it is interesting to compare populations on both sides of this strait.

Here, we examined mitochondrial DNA (mtDNA) control region sequences of a large sample of loggerhead turtles collected from different areas of the Tunisian coast in order to (1) assess genetic structuring along the Tunisian coast, (2) elucidate the natal origins of turtles foraging in Tunisian feeding grounds, and (3) improve our understanding of factors impacting dispersal in the central Mediterranean region.

\section{MATERIALS AND METHODS}

\section{Sample collection}

Samples were collected from 175 loggerhead turtles which washed ashore $(\mathrm{n}=129)$ or were captured incidentally $(\mathrm{n}=46)$ along the Tunisian coast (Fig. 1). Sampling was carried out throughout the year over a $6 \mathrm{yr}$ period from 2004 to $2009\left(\mathrm{n}_{2004}=12 ; \mathrm{n}_{2005}=10\right.$; $\left.\mathrm{n}_{2006}=20 ; \mathrm{n}_{2007}=95 ; \mathrm{n}_{2008}=33 ; \mathrm{n}_{2009}=5\right)$. For each specimen, the curved carapace length (CCL) was measured using a flexible tape meter to the nearest $\mathrm{cm}$. Blood was taken from the dorsal cervical sinus of live turtles, following the procedures of Owens \& Ruiz (1980), and stored at $-20^{\circ} \mathrm{C}$. Live specimens, which were rehabilitated in the Sea Turtle Rescue Center of the 'Institut National des Sciences et Technologies de la Mer' (INSTM) at Monastir, were tagged before release to avoid resampling. Muscle or skin samples were collected from dead animals and preserved in $70 \%$ ethanol.

\section{Laboratory methods}

Whole genomic DNA was isolated by standard phenol chloroform extraction (Hillis et al. 1996). A 510 base pair (bp) fragment of the mtDNA control region was amplified by polymerase chain reaction (PCR) using the primers L71 and H599 (Laurent et al. 1998). The PCR protocol was $94^{\circ} \mathrm{C}$ for $5 \mathrm{~min}$, followed by 35 cycles at $94^{\circ} \mathrm{C}$ for $1 \mathrm{~min}, 56^{\circ} \mathrm{C}$ for $1 \mathrm{~min}$ and $72^{\circ} \mathrm{C}$ for $1 \mathrm{~min}$, with a final extension at $72^{\circ} \mathrm{C}$ for 10 min (Carreras et al. 2006). Both positive and negative controls were run to ensure that there was no contamination of the DNA samples. PCR products were visualized in a $1 \%$ agarose gel and then purified using a Qiagen purification kit following the manufacturer's instructions. Purified products were sequenced on an automated sequencer (Applied Biosystems model 3700 or 3730 ), and sequences were corrected and aligned using the BioEdit Sequence Alignment Editor v.7.0.9.0 (Hall 1999) and ClustalW programme (Chenna et al. 2003).

\section{Data analysis}

Differences in the body size of specimens from the 3 areas were evaluated using a Kruskal-Wallis test followed by a post hoc test using the software SPSS 18.0 (SPSS Inc. 2010). In order to avoid confounding the local nesting population with resident foraging turtles, individuals of adult size which might have been transient breeders moving through the area were removed (Limpus \& Reed 1985). Therefore, turtles with $\mathrm{CCL} \geq 66.5 \mathrm{~cm}$, i.e. the minimum CCL of a nesting female loggerhead in the Mediterranean (Margaritoulis et al. 2003) sampled during mating and nesting seasons, were not considered in the analysis.

MtDNA sequences were compared with previously described $380 \mathrm{bp}$ loggerhead haplotypes published in 
the Archie Carr Center for Sea Turtle Research DNA database (ACCSTR; http://accstr.ufl.edu). Genetic diversity of the samples was estimated by haplotype diversity ( $h$; Nei 1987) and nucleotide diversity $(\pi ;$ Tajima 1983). Genetic differentiation between sampling locations was assessed using pairwise $F_{\mathrm{ST}}$ computations based on haplotype frequencies (Weir \& Cockerham 1984), and the exact test of population differentiation (Raymond \& Rousset 1995). As exact origins of stranded turtles could not be determined precisely, a test of homogeneity was run within regions by comparing captured and stranded samples. We then tested for genetic differentiation between regions; first, we considered all of the captured and stranded specimens; second, we excluded stranded turtles. Annual variation was assessed within each region. Our samples were also compared with previously studied foraging grounds in the Mediterranean Sea and the Atlantic Ocean: eastern and western Italy, northeastern Spain, the Balearic Islands of Pitiüses and Gimnesies (Carreras et al. 2006), eastern and western oceanic Mediterranean zones, Egypt, Tunisia (Laurent et al. 1998), Lampedusa (Casale et al. 2008b), the Alboran Sea, Gulf of Cadiz (Revelles et al. 2007b) and north-central Adriatic (Giovannotti et al. 2010). All computations were carried out by the program Arlequin v.3.0. (Excoffier et al. 2005). Correction for multiple tests was performed using a modified false discovery rate (FDR) procedure (Narum 2006).

Natal origins of loggerheads foraging in Tunisian waters were determined using a Bayesian mixed stock analysis (MSA) based on the many-to-many approach (Bolker et al. 2007) using the software WinBugs (Spiegelhalter et al. 2004). The results can be expressed as the proportions of individuals in each foraging ground contributed by different rookeries in a 'foraging-ground-centric' way (i.e. the proportion of individuals from each rookery in each foraging ground) or in a 'rookery-centric' way (i.e. the proportion of individuals in each rookery moving to each foraging ground). This method is not biased by the presence of rare haplotypes, which are regularly observed in sea turtle populations, and allows the incorporation of environmental data into the analysis as well as ecological data, such as source population size (Bolker et al. 2007 and references therein). Convergence of chains to the desired posterior density was checked to test against anomalous real- izations of distributions. An estimated shrink factor (Gelman \& Rubin 1992) close to 1 indicated convergence, and acceptable values were $<1.2$ (Kass et al. 1998). The baseline sample was obtained from published data. For the Atlantic stock, we considered the following reproductive units: south Florida, northeast Florida-North Carolina, northwest Florida, Dry Tortugas (USA), Mexico (Quintana Roo, Yucatan) (Encalada et al. 1998, Bowen et al. 2005), Brazil units (BR1: Rio de Janeiro/Espirito Santo, BR2: Bahia/Sergipe; Reis et al. 2010) and Cape Verde Islands (Monzon-Arguello et al. 2010; our Fig. 2). Nesting population size data for rookeries were obtained from Ehrhart et al. (2003) and Monzon-Arguello et al. (2010). The Mediterranean rookeries considered in the study were Greece (Zakynthos, Kyparissia, Lakonikos and Crete), Turkey, Cyprus, Israel (Encalada et al. 1998, Laurent et al. 1998, Carreras et al. 2007), and Italy (Garofalo et al. 2009; our Fig. 2). The population size of these units, estimated as the annual average number of nests for these units, were obtained from Mingozzi et al. (2007) for Italy, Margaritoulis et al. (2003) for Turkey and Casale \& Margaritoulis (2010) for the others (Table 1).

\section{RESULTS}

The 175 individuals sampled were assigned to 1 of the 3 regions based on the location where they were found. After excluding possible breeders, only 151 turtles with CCL ranging from 18.5 to $81 \mathrm{~cm}$ were considered for the analyses (Table 2$)$ : NTC $\left(\mathrm{n}_{\text {captured }}=\right.$ $\left.11 ; \mathrm{n}_{\text {stranded }}=6\right)$, GHAM $\left(\mathrm{n}_{\text {captured }}=24 ; \mathrm{n}_{\text {stranded }}=12\right)$ and GGAB $\left(\mathrm{n}_{\text {captured }}=27 ; \mathrm{n}_{\text {stranded }}=71\right)$.

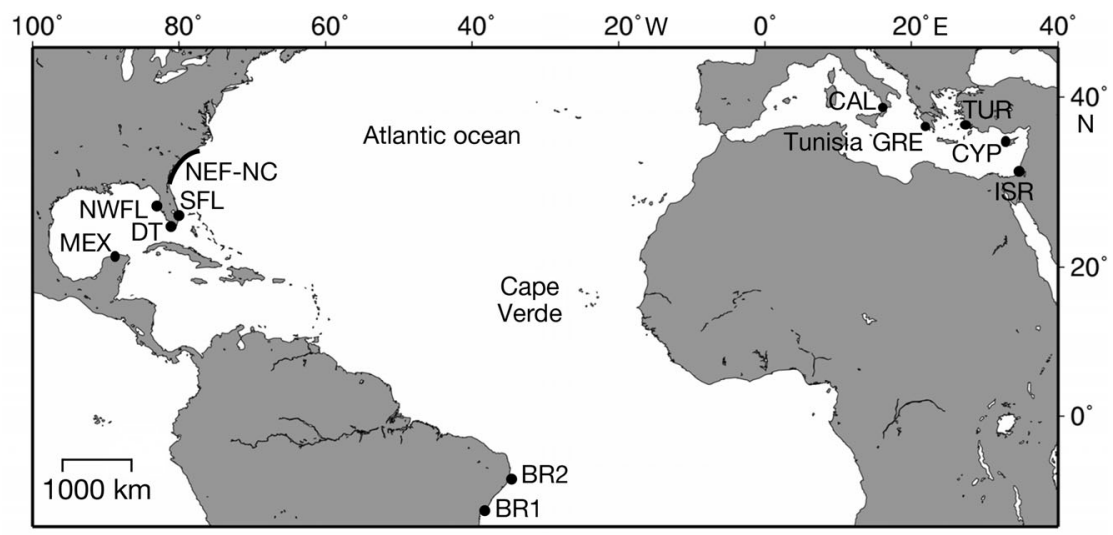

Fig. 2. Caretta caretta. Atlantic Ocean and Mediterranean Sea, showing locations of nesting populations used in the mixed stock analysis (see references in Table 1). NEF-NC: northeast Florida-North Carolina; SFL: south Florida; NWFL: northwest Florida; DT: Dry Tortugas; MEX: Mexico; BR1: Rio de Janeiro/Espirito Santo, BR2: Bahia/Sergipe; CAL: Calabria (Italy); GRE: Greece; TUR: Turkey; CYP: Cyprus; ISR: Israel 
Table 1. Caretta caretta. Haplotype distribution in the Mediterranean and Atlantic nesting populations used in the mixed stock analysis. Data on population size (pop. size; the annual average number of nests) are derived from Ehrhart et al. (2003), Margaritoulis et al. (2003), Mingozzi et al. (2007), Casale \& Margaritoulis (2010) and Monzon-Arguello et al. (2010). N: sample size; NEF-NC: northeast Florida-North Carolina; NWFL: northwest Florida; BR1: Rio de Janeiro/Espirito Santo, BR2: Bahia/Sergipe

\begin{tabular}{|c|c|c|c|c|c|c|c|c|c|c|c|c|c|c|c|c|c|c|c|c|c|c|}
\hline Source pop. & $\mathrm{N}$ & 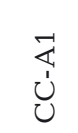 & 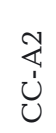 & $\underset{U}{\stackrel{Z}{4}}$ & 莣 & $\sum_{0}^{\infty}$ & $\begin{array}{l}\sum_{u}^{0} \\
U_{u}^{\prime}\end{array}$ & 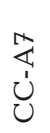 & $\bigcup_{U}^{\infty}$ & 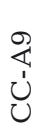 & 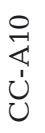 & 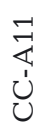 & 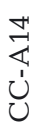 & 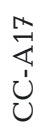 & 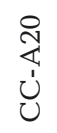 & $\underset{U}{\stackrel{Z}{J}}$ & $\underset{ن}{\stackrel{0}{\mathbb{C}}}$ & $\underset{\cup}{\stackrel{S}{i}}$ & 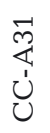 & 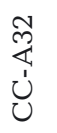 & $\underbrace{\stackrel{N}{\sharp}}_{U}$ & $\begin{array}{l}\text { Pop. } \\
\text { size }\end{array}$ \\
\hline \multicolumn{23}{|l|}{ Atlantic } \\
\hline South Florida ${ }^{a, b}$ & 109 & 52 & 45 & 4 & - & 1 & - & 3 & - & - & - & 1 & 2 & - & 1 & - & - & - & - & - & - & 67100 \\
\hline NEF-NC ${ }^{a, b}$ & 105 & 104 & 1 & - & - & - & - & - & - & - & - & - & - & - & - & - & - & - & - & - & - & 6200 \\
\hline $\mathrm{NWFL}^{\mathrm{a}, \mathrm{b}}$ & 49 & 38 & 7 & 2 & - & - & - & 2 & - & - & - & - & - & - & - & - & - & - & - & - & - & 600 \\
\hline Mexico $^{\mathrm{a}, \mathrm{b}}$ & 20 & - & 11 & 2 & - & - & - & - & 1 & 1 & 5 & - & - & - & - & - & - & - & - & - & - & 1800 \\
\hline Cape Verde $e^{c}$ & 186 & 127 & 2 & - & - & - & - & - & - & - & - & 1 & - & 53 & - & - & - & - & - & - & 3 & 14000 \\
\hline $\mathrm{BR}^{\mathrm{d}}$ & 114 & - & - & - & 113 & - & - & - & - & - & - & - & - & - & - & - & 1 & - & - & - & - & 1858 \\
\hline $\mathrm{BR} 2^{\mathrm{d}}$ & 76 & - & - & - & 63 & - & - & - & - & - & - & - & - & - & - & 13 & - & - & - & - & - & 2676 \\
\hline Dry Tortugas $^{\mathrm{a}, \mathrm{b}}$ & 58 & 4 & 50 & - & - & - & - & - & - & 2 & 2 & - & - & - & - & - & - & - & - & - & - & 217 \\
\hline \multicolumn{23}{|l|}{ Mediterranean } \\
\hline Greece $^{\mathrm{a}, \mathrm{e}, \mathrm{f}}$ & 89 & - & 82 & - & - & - & 5 & - & - & - & 1 & - & - & - & - & - & - & - & - & 1 & - & 3472 \\
\hline Turkey & 32 & - & 19 & 13 & - & - & - & - & - & - & - & - & - & - & - & - & - & - & - & - & - & 1366 \\
\hline Cyprus $^{\mathrm{e}}$ & 35 & - & 35 & - & - & - & - & - & - & - & - & - & - & - & - & - & - & - & - & - & - & 694 \\
\hline Israel $^{\mathrm{f}}$ & 20 & - & 17 & - & - & - & - & - & - & - & - & - & - & - & - & - & - & 3 & - & - & - & 57 \\
\hline Calabria (Italy) $^{\mathrm{g}}$ & 38 & - & 22 & - & - & - & - & - & - & - & - & - & - & - & 14 & - & - & - & 2 & - & - & $15-20$ \\
\hline
\end{tabular}

Table 2. Caretta caretta. Mitochondrial DNA haplotype distribution in the Mediterranean and Atlantic foraging populations. N: sample size; WIT: western Italy; EIT: eastern Italy; LAM: Lampedusa; GIM: Gimnesies; PIT: Pitiüses; NES; northeastern Spain; WMED: western Mediterranean oceanic zone; EMED: eastern Mediterranean oceanic zone; ALB: Alboran Sea; CAD: Gulf of Cadiz; NCA: north central Adriatic; TUN: Tunisia; EGPT: Egypt; NTC: northern Tunisian coast; GHAM: Gulf of Hammamet; GGAB: Gulf of Gabès. CCL: curved carapace length; values in brackets show length ranges; asterisk shows straight carapace length (SCL) values transformed to CCL according to the equation CCL $=1.388+(1.053) \times$ SCL $($ Bjorndal et al. 2000)

\begin{tabular}{|c|c|c|c|c|c|c|c|c|c|c|c|c|c|c|c|c|c|c|c|c|c|c|c|c|}
\hline $\begin{array}{l}\text { Foraging } \\
\text { grounds }\end{array}$ & $\mathrm{N}$ & 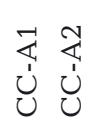 & 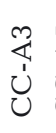 & 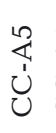 & 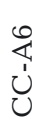 & 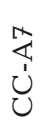 & \begin{tabular}{l}
0 \\
\multirow{4}{4}{} \\
$ن$
\end{tabular} & 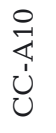 & 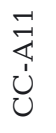 & 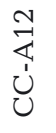 & 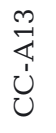 & $\underset{U}{\stackrel{Z}{⿺}}$ & 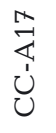 & 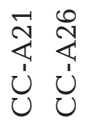 & $\bigcup_{0}^{\stackrel{N}{i}}$ & 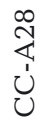 & 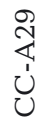 & 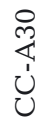 & $\begin{array}{l}\overrightarrow{2} \\
\dot{U} \\
\dot{U}\end{array}$ & 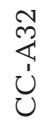 & 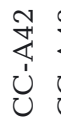 & 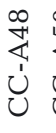 & 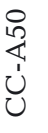 & CCL $(\mathrm{cm})$ \\
\hline WIT $^{\mathrm{a}}$ & 49 & 440 & 3 & - & - & - & - & - & - & - & - & - & - & -1 & - & - & - & - & - & 1 & - & - & - & $49.7 \pm 13.7(25.0-72.0)$ \\
\hline $\mathrm{EIT}^{\mathrm{a}}$ & 58 & 248 & 5 & - & - & - & - & - & - & - & - & - & - & -1 & - & - & - & - & 1 & 1 & - & - & - & $36.8 \pm 12.5(16.0-65.0)$ \\
\hline $\mathrm{LAM}^{\mathrm{b}}$ & 70 & 460 & 4 & - & - & - & - & - & - & - & - & - & - & -1 & - & - & - & - & - & - & - & - & 1 & $58.8(36.5-85.0)$ \\
\hline GIM $^{\mathrm{a}}$ & 31 & 1611 & 3 & - & - & - & - & - & - & - & - & - & - & $-\quad-$ & 1 & - & - & - & - & - & - & - & - & $50.0 \pm 9.6(32.0-73.0)$ \\
\hline $\mathrm{PIT}^{\mathrm{a}}$ & 32 & 917 & - & 1 & - & - & 1 & - & - & - & 1 & 2 & - & -1 & - & - & - & - & - & - & - & - & - & $49.6 \pm 9.0(28.0-66.0)$ \\
\hline $\mathrm{NES}^{\mathrm{a}}$ & 112 & 1481 & 8 & 1 & - & 1 & - & 1 & - & - & - & - & - & -2 & - & 1 & 2 & 1 & - & - & - & - & - & $44.1 \pm 14.9(20.0-100.0)$ \\
\hline $\mathrm{WMED}^{\mathrm{c}}$ & 59 & 1346 & - & - & - & - & - & - & - & - & - & - & - & $-\quad-$ & - & - & - & - & - & - & - & - & - & $47.4 \pm 10.4(27.6-69.0)$ \\
\hline EMED $^{\mathrm{c}}$ & 52 & 1232 & 3 & - & - & 1 & 1 & - & - & - & 1 & - & - & -2 & - & - & - & - & - & - & - & - & - & $45.9 \pm 7.5(33.0-75.5)$ \\
\hline $\mathrm{ALB}^{\mathrm{d}}$ & 65 & 2631 & 2 & - & - & - & - & 1 & 2 & - & - & 1 & - & $1-$ & - & - & - & - & - & - & 1 & - & - & $46.7^{*}(17.2-84.6)$ \\
\hline $\mathrm{CAD}^{\mathrm{d}}$ & 40 & 1915 & - & - & - & - & 2 & - & - & 1 & - & 1 & 1 & $1-$ & - & - & - & - & - & - & - & - & - & $42.4^{*}(15.1-81.4)$ \\
\hline $\mathrm{NCA}^{\mathrm{e}}$ & 65 & -57 & 6 & - & 2 & - & - & - & - & - & - & - & - & $-\quad-$ & - & - & - & - & - & - & - & - & - & $(15.1-89.8)^{*}$ \\
\hline $\mathrm{TUN}^{\mathrm{c}}$ & 34 & -33 & - & - & - & - & - & - & - & - & - & - & - & -1 & - & - & - & - & - & - & - & - & - & $61.0 \pm 15.1(32.3-91.8)$ \\
\hline $\mathrm{EGPT}^{\mathrm{c}}$ & 23 & -18 & 2 & - & - & - & - & - & - & - & - & - & - & -2 & - & - & - & - & - & - & - & 1 & - & $67.0 \pm 9.5(49.4-86.3)$ \\
\hline NTC $^{f}$ & 17 & 510 & - & - & 1 & - & - & 1 & - & - & - & - & - & $-\quad-$ & - & - & - & - & - & - & - & - & - & $58.2 \pm 10.9(35.0-70.0)$ \\
\hline GHAM $^{\mathrm{f}}$ & 36 & 230 & 2 & - & 2 & - & - & - & - & - & - & - & - & $-\quad-$ & - & - & - & - & - & - & - & - & - & $45.7 \pm 16.4(18.5-75.1)$ \\
\hline $\mathrm{GGAB}^{\mathrm{f}}$ & 98 & -84 & 9 & - & 1 & - & - & 1 & - & - & - & - & - & -2 & - & 1 & - & - & - & - & - & - & - & $56.1 \pm 9.5(29.5-81.0)$ \\
\hline
\end{tabular}


Loggerhead turtle specimens from the GHAM were significantly smaller than those from the NTC $(p=0.002)$ and the GGAB $(p=0.000)$. CCL means were respectively $45.7 \pm 16.4,58.2 \pm 10.9$ and $56.1 \pm$ $9.5 \mathrm{~cm}$ (Table 2). Seven distinct haplotypes were detected: CC-A1, CC-A2, CC-A3, CC-A6, CC-A10, CC-A26 and CC-A28 (Table 2). The endemic Atlantic haplotype CC-A1 was detected only in the NTC $(29.4 \%)$ and in the GHAM (5.5\%). Haplotypes CC-A2, CC-A3 and CC-A10 are shared between Mediterranean and Atlantic nesting populations, although the first 2 occur at much higher frequencies in the Mediterranean region (Table 1). Haplotype CC-A6 has been reported only from Greek nesting beaches (Table 1). Haplotypes CC-A26 and CC-A28 could not be assigned to a rookery of origin, but they have previously been reported from both neritic and oceanic Mediterranean foraging grounds (see references in Table 2). These 'orphan' haplotypes were excluded from the MSA because they do not provide any information about the contributions of nesting populations to feeding grounds.

No genetic differentiation was found between captured and stranded samples in each study area. Similarly, within-region comparisons of the largest samples from 2006, 2007 and 2008 revealed no annual variation. Thus, all samples (stranded and captured during the $6 \mathrm{yr}$ period) were pooled for comparison between the studied areas. A significant genetic differentiation was found between the GGAB and the NTC; neither differed significantly from the GHAM (Table 3). The same result was obtained when only captured turtles were considered. The NTC population was genetically homogenous with the Atlantic foraging population of Cadiz and the Mediterranean ones from the Balearic Islands, northeast Spain, the eastern and the western oceanic Mediterranean zones and the Alboran Sea (see references in Table 2), all characterized by a high frequency of the CC-A1 haplotype (Fig. 3, Table 2). The GGAB exhibited genetic differences with all of the foraging grounds, except with the north-central Adriatic, Lampedusa and eastern and western Italy. It also showed one of the lowest genetic diversities (Table 3).

The 'foraging-ground-centric' many-to-many results suggested a high contribution from the Atlantic stocks to the Tunisian foraging grounds that decreases rapidly from the north to the south (total contribution, NTC: $97.1 \pm 11.9 \%$; GHAM: $57.8 \pm 4.4 \%$; GGAB: $17.9 \pm 1.6 \%$ ). The proportions of Mediterranean contributions also vary gradually, but in the opposite direction (GGAB: $82.1 \pm$

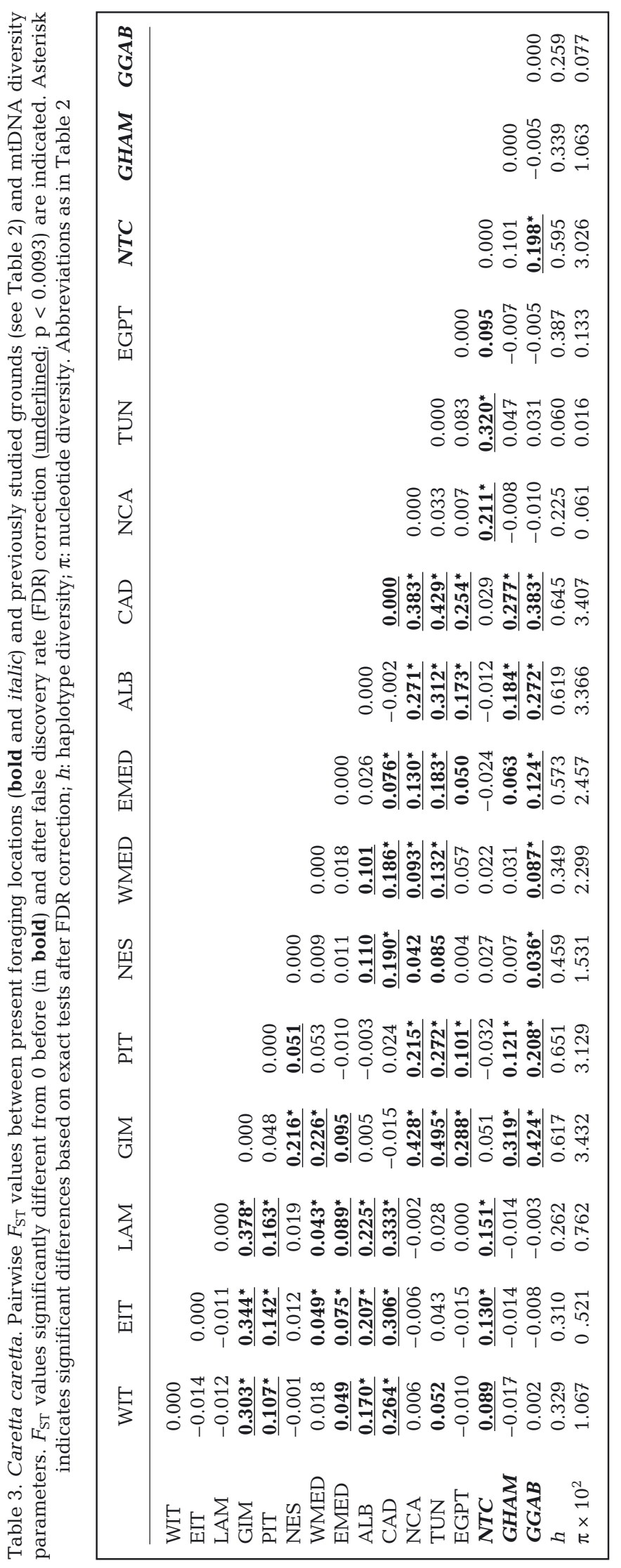




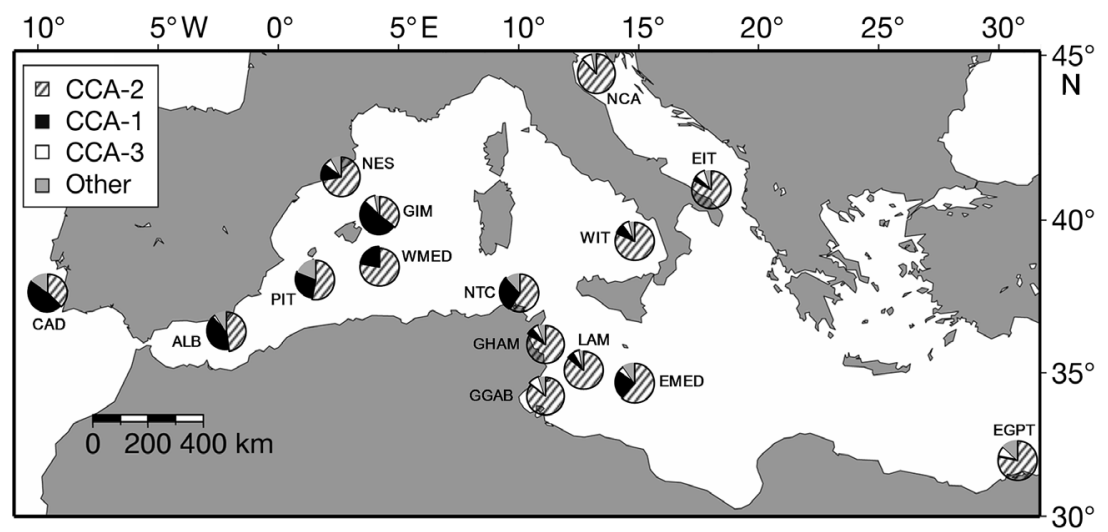

Fig. 3. Caretta caretta. Mediterranean region, showing mtDNA haplotype frequencies in the studied areas and in other previously studied foraging grounds (see references in Table 2). WIT: western Italy; EIT: eastern Italy; LAM: Lampedusa; GIM: Gimnesies; PIT: Pitiüses; NES; northeastern Spain; WMED: western Mediterranean oceanic zone; EMED: eastern Mediterranean oceanic zone; ALB: Alboran Sea; CAD: Gulf of Cadiz; NCA: north central Adriatic; NTC: northern Tunisian coast; GHAM: Gulf of Hammamet; GGAB: Gulf of Gabès; EGPT: Egypt

$16.2 \%$; GHAM: $42.2 \pm 8.7 \%$; NTC: $2.9 \pm 0.4 \%$ ). The south Florida rookery was the main Atlantic contributor to the studied areas, while the main Mediterranean contribution came from Greek rookeries (Table 4).

Table 4. Caretta caretta. 'Foraging-ground centric' estimated contributions (mean $\pm \mathrm{SD}$ ) of the Atlantic and Mediterranean rookeries to the Tunisian foraging populations (NTC: northern Tunisian coast; GHAM: Gulf of Hammamet; GGAB: Gulf of Gabès). SFL: south Florida; NEF-NC: northeast FloridaNorth Carolina; NWFL: northwest Florida; MEX: Mexico; CV: Cape Verde; BR1: Rio de Janeiro/Espirito Santo; BR2: Bahia/Sergippe; DT: Dry Tortugas; GRE: Greece; TUR:

Turkey; CYP: Cyprus; ISR: Israel; CAL: Calabria (Italy)

\begin{tabular}{|lccc|}
\hline & NTC & GHAM & GGAB \\
\hline Atlantic & & & \\
SFL & $73.0 \pm 75.7$ & $34.5 \pm 20.0$ & $2.6 \pm 1.8$ \\
NEF-NC & $7.0 \pm 5.8$ & $3.9 \pm 2.8$ & $1.1 \pm 0.7$ \\
NWFL & $0.7 \pm 0.5$ & $2.7 \pm 1.7$ & $1.1 \pm 0.8$ \\
MEX & $2.4 \pm 1.8$ & $7.0 \pm 4.5$ & $7.8 \pm 5.6$ \\
CV & $9.2 \pm 7.1$ & $3.7 \pm 2.7$ & $1.1 \pm 0.7$ \\
BR1 & $2.0 \pm 1.6$ & $2.2 \pm 1.5$ & $1.0 \pm 0.7$ \\
BR2 & $2.7 \pm 2.3$ & $2.5 \pm 1.7$ & $1.0 \pm 0.7$ \\
DT & $0.2 \pm 0.1$ & $1.3 \pm 0.8$ & $2.2 \pm 1.7$ \\
Total & $97.1 \pm 11.9$ & $57.8 \pm 4.4$ & $17.9 \pm 1.6$ \\
Mediterranean & & & \\
GRE & $1.7 \pm 1.1$ & $30.9 \pm 35.3$ & $47.5 \pm 48.9$ \\
TUR & $0.6 \pm 0.4$ & $7.0 \pm 5.9$ & $23.0 \pm 22.1$ \\
CYP & $0.4 \pm 0.3$ & $3.9 \pm 2.2$ & $10.8 \pm 9.4$ \\
ISR & $0.0 \pm 0.0$ & $0.2 \pm 0.1$ & $0.4 \pm 0.3$ \\
CAL & $0.0 \pm 0.0$ & $0.2 \pm 0.1$ & $0.4 \pm 0.3$ \\
Total & $2.9 \pm 0.4$ & $42.2 \pm 8.7$ & $82.1 \pm 16.2$ \\
\hline
\end{tabular}

\section{DISCUSSION}

We demonstrated for the first time the presence of a particular pattern of genetic variation among the loggerhead turtle foraging populations in the different Tunisian neritic habitats. The extreme samples (NTC and GGAB) differed genetically from each other, while both were similar to the central one (GHAM). This genetic structure was mainly due to the gradual variation of the Atlantic haplotype CCA1 frequency among the 3 areas. This was highest in the NTC, intermediate in the GHAM and null in the GGAB. This finding was confirmed by the MSA estimates, which suggested that the proportions of Atlantic turtles were very high in the NTC and decreased southward, while the proportions of Mediterranean contributions were higher in the GGAB and decreased in the opposite direction. A potential source of uncertainty about the observed pattern of genetic structuring is the reduced sample size in the NTC compared to both other samples and the possible existence of temporal variations in the haplotype frequencies that may lead to erroneous differences between the sampling areas. Based on our results and previous findings, these artefacts should not be considered a major source of concern. First, we showed no annual variation within each region when comparing samples from 3 successive years (2006-2008) representing more than $80 \%$ of the total sample. In the same way, comparison of our samples and those previously analysed in the same region by Casale et al. (2008b) and Laurent et al. (1998) suggested no significant differences. Bass et al. (2004) found no annual variation in the genetic composition of a foraging ground in North Carolina, USA, during a 3 yr period and suggested that temporal variation may occur at a much larger time scale. Second, the reduced sample size in the NTC may have not completely uncovered the mtDNA haplotype diversity in the area, but was sufficient to reveal particularities in the haplotype composition that could not be due only to chance. In fact, in the NTC sample we found 5 turtles carrying the endemic Atlantic haplotype CC-A1, while in the GHAM and GGAB samples which were respectively 2 and 6 times larger, only 2 and 0 CC-A1 turtles were detected. These results indicate that the genetic com- 
position was non-homogeneous and the distribution of foraging turtles was non-random among the 3 areas.

The observed pattern of genetic variation could be generated by phenomena such as sea surface currents and habitat characteristics that affect the mixing of turtles when reaching foraging grounds. This would have a noticeable effect on the genetic makeup of foraging aggregations, especially when turtles come from reproductive units as different as Mediterranean and Atlantic ones (Carreras et al. 2006, 2007, 2011). Atlantic water penetrates the Mediterranean Sea through the Strait of Gibraltar and forms the Algerian current (AC) which may carry Atlantic juvenile loggerheads eastwards along the African coast to the NTC. The current in the NTC flows close to the coastline, where the continental shelf is reduced and the adjoining neritic habitats are characterized by rocky bottoms and relatively deep waters. Therefore, Atlantic individuals may occasionally enter this area although they do not actually recruit to the neritic stage. At the Siculo-Tunisian Channel, the AC divides into 2 main streams, the Ionian (Atlantic Ionian Stream, AIS), which flows eastwards in the northern Ionian Sea, and the Atlantic Tunisian Current (ATC), which follows the $200 \mathrm{~m}$ isobath (Onken et al. 2003, Béranger et al. 2004). The latter current may carry Atlantic turtles in close proximity to the GHAM, which coincides with the beginning of the African continental shelf. The very large extension of the continental shelf in the southern zone of Tunisia may divert Atlantic juveniles, carried by the ATC, from the Tunisian coast preventing them from entering the GGAB. It is likely that these turtles rarely recruit to this gulf because of its topography and distance from the ATC. The gulf is, $100 \mathrm{~km}$ long and $100 \mathrm{~km}$ wide, its depths typically range from 20 to $50 \mathrm{~m}$, and the ATC flows over the Tunisian slope located at about $250 \mathrm{~km}$ from its coast (Fig. 1) (Onken et al. 2003, Béranger et al. 2004). Similarly, it has been shown that Atlantic loggerheads in the western Mediterranean region avoid the European continental shelf and remain linked to Atlantic water masses transported by the AC along the African coast (Cardona et al. 2005, Carreras et al. 2006, Revelles et al. 2007b). The sea surface current in the studied area does not likely represent an effective obstacle to such good swimmers but probably acts as a supplementary factor facilitating their passage to deeper waters where the food quality is presumably more suitable to their oceanic developmental stage and/or where the predation risk is probably lower (Bolten 2003).
MSA results showed that a high contribution from the Atlantic rookeries was detected in the NTC and the GHAM. The presence of Atlantic turtles in shallow Tunisian foraging areas not far from the oceanic foraging areas in the Balearic and the Ionian Seas could be explained by opportunistic behaviour of oceanic-stage Atlantic turtles. These turtles feed mainly on pelagic prey but also occasionally switch to feeding on benthic prey. Previous studies have suggested that immature turtles do not recruit to the benthic phase in 1 step from the strict oceanic pelagic phase but progressively from a later flexible neritic stage (Laurent et al. 1998, Eckert et al. 2008). In a recent ecological study of foraging turtles in the central Mediterranean, Casale et al. (2008a) suggested that this behavioural flexibility is a general feature and proposed a relaxed ontogenetic model of life history for the species. Our data support this model, as we noticed the presence of small juveniles ( $\mathrm{n}=10, \mathrm{CCL}<30 \mathrm{~cm}$ ) in the eastern and southern regions used by both Mediterranean and Atlantic turtles. We showed a relatively high contribution from some Atlantic rookeries, such as the Mexican one, particularly to the GHAM and GGAB (Table 4). It is likely that this contribution was overestimated and could be attributed to similarities of haplotype frequency patterns observed between the areas, rather than a real connectivity that has never been reported in the literature. Analysing longer fragments of the mtDNA, which makes it possible to uncover genetic differentiation between locations sharing the same short fragments, would improve the resolution of the MSA technique (Abreu-Grobois et al. 2006).

Different sizes of Atlantic CC-A1 individuals were detected in the NTC and the GHAM, ranging from 23.5 to $70.0 \mathrm{~cm}$. We detected the smallest CC-A1 turtle in the Mediterranean basin, which suggests that Atlantic turtles may frequent Mediterranean zones at earlier developmental stages than previously thought $(\mathrm{CCL}=29.7 \mathrm{~cm}$; Laurent et al. 1998). Hays \& Marsh (1997) reported that the modal straight carapace length of juvenile turtles crossing the Atlantic from the northern American beaches to the coast of the UK, at about the same longitude as the western border of the Mediterranean, was $20.5 \mathrm{~cm}(\approx 23.0 \mathrm{~cm}$ CCL), corresponding to an estimated modal age between 1.8 and 3.7 yr (Hays \& Marsh 1997). It therefore seems that after achieving their trans-Atlantic migration, some Atlantic early juveniles could rapidly reach remote Mediterranean foraging areas to take advantage of the abundant food resources there. 
The MSA estimates showed that the 3 main Mediterranean nesting rookeries contribute significantly to the GGAB, with the highest contribution from Greece, followed by Turkey and Cyprus. The majority of turtles inhabiting the gulf are in the high size classes $(83 \%$ of CCLs $>50 \mathrm{~cm})$, composed mainly of late juveniles and sub-adults. Our results confirm the importance of the gulf for the entire Mediterranean loggerhead turtle population already suggested by previous mark and recapture studies (Margaritoulis et al. 2003) and satellite tracking telemetry surveys (Broderick et al. 2007, Zbinden et al. 2007, 2011, Hays et al. 2010). The large continental shelf, the richness of the sandy bottom and the warm water during the winter made this area one of the most important foraging grounds for neritic-stage loggerhead turtles in the Mediterranean Sea. Other contributions from indeterminate origins were noted, as individuals carrying unidentified haplotypes CC-A26 and CCA28 were detected. These haplotypes, detected exclusively in Mediterranean foraging grounds (Laurent et al. 1998, Carreras et al. 2006) could be of Mediterranean origin. As the most important Mediterranean rookeries are relatively well characterized, these haplotypes likely come from nesting sites not yet analysed, such as the large Libyan site, or from other rookeries that were poorly sampled. On the other hand, the very low contributions from the Italian and the Israeli rookeries estimated by MSA and the corresponding absence of their respective endemic haplotypes CC-A20, CC-A31 and CC-A29 (Table 1) are likely explained by the very small size of these 2 potential sources and do not necessarily relate to a low connectivity. Recent satellite tracking projects launched in both countries (TARTACare Calabria, Italy, A.T. Mingozzi pers. comm.; and the Israel Sea Turtle Tracking Project, Y. Levy pers. comm., www.seaturtle.org/tracking/project), showed migration of several nesting females toward the GGAB. This highlights the importance of the information gathered from field studies for a better understanding of the migratory connectivity in such species.

In conclusion, our results suggested a non-random distribution of foraging turtles along the Tunisian coast in light of the gradual variation in haplotype composition among the 3 studied areas. The genetic structuring observed over $1300 \mathrm{~km}$ of a continuous coastline was probably driven by non-apparent barriers such as sea surface currents and geomorphology. Nevertheless, the observed pattern of haplotype distribution should not be considered static since it depends on the relative contribution from source populations and temporal variations in the reproduc- tive success at each rookery. We showed for the first time that the NTC hosts a high proportion of Atlantic turtles. This could be related to the geographical position of this area representing an obligatory passage for Atlantic turtles moving between both Mediterranean basins and probably represents a foraging zone utilized mainly by Atlantic stocks. Further ecological, behavioural and genetic investigations could help resolve this issue.

Overall, the Tunisian coast is frequented by turtles from both Atlantic and Mediterranean stocks and thus seems to represent a hotspot for conservation strategies. Particular concern arises from the intense anthropogenic activities in this region that pose a threat for both Atlantic and Mediterranean populations. Conservation models show that adult and large immature turtles make the greatest contribution to the survival of a population (Crouse et al. 1987, Laurent et al. 1998); hence, special efforts should be made to reduce turtle bycatch when implementing future management strategies. The GGAB, which is among the areas most exploited by fisheries in the Mediterranean Sea, interestingly hosts a much higher proportion of Mediterranean turtles. Given that the stock of turtles nesting in the Mediterranean is much more depleted than that in the Atlantic rookeries (Ehrhart et al. 2003), a reduction in turtle bycatch and mortality in this area should be considered a priority to protect the Mediterranean population.

Acknowledgements. We thank P. Casale and 4 anonymous reviewers for their helpful comments on earlier versions of the manuscript. We are also grateful to A.T. Mingozzi and P. Luschi, and to Y. Levy for giving us permission to refer to the 'TARTACare Calabria, Italy' and 'Israel Sea Turtle Tracking Project' tracking projects, respectively. We acknowledge use of the Maptool program (www.seaturtle.org) for the drawing of the figures.

\section{LITERATURE CITED}

Abreu-Grobois A, Horrocks J, Formia A, Dutton DL and others (2006) New mtDNA D-loop primers which work for a variety of marine turtle species may increase the resolution of mixed stock analysis. In: Frick M, Panagopoulous A, Rees A, William K (eds) 26th Annu Symp Sea Turtle Biol Conserv. International Sea Turtle Society, Athens, p 179

Bass AL, Epperly SP, Braun-McNeill J (2004) Multi-year analysis of stock composition of the loggerhead turtle (Caretta caretta) foraging habitat using maximum likelihood and Bayesian methods. Conserv Genet 5:783-796

Ben Mustapha K, Afli A, Hattour A, El Abed A (2003) Sessile megabenthic species from Tunisian littoral sites. In: Levi D, Bahri T, Camilleri M, Jarboui O and others (eds) Report of the expert consultation on the spatial distribution of demersal resources in the straits of Sicily and the 
influence of environmental factors and fishery characteristics. MedSudMed Tech Doc 2. FAO, Mazara del Vallo, p 82-97

Ben Salem S, Franquesa R, El Abed A (2002) Indicateurs socioéconomiques pour la pêche au Golfe de Gabès (Tunisie). Étude de cas. FAO-COPEMED, Salerno

Bentivegna F (2002) Intra-Mediterranean migration of loggerhead sea turtles monitored by satellite telemetry. Mar Biol 141:795-800

Béranger K, Mortiera L, Gasparini GP, Gervasio L, Astraldi M, Crépon M (2004) The dynamics of the Sicily Strait: a comprehensive study from observations and models. Deep-Sea Res II 51:411-440

Bjorndal KA, Bolten AB, Martins HR (2000) Somatic growth model of juvenile loggerhead sea turtles Caretta caretta: duration of pelagic stage. Mar Ecol Prog Ser 202:265-272

Bolker BM, Okuyama T, Bjorndal KA, Bolten AB (2007) Incorporating multiple mixed stocks in mixed stock analysis: 'many-to-many' analyses. Mol Ecol 16:685-695

Bolten AB (2003) Active swimmers - passive drifters: the oceanic juvenile stage of loggerheads in the Atlantic system. In: Bolten AB, Witherington B (eds) Loggerhead sea turtles. Smithsonian Institution, Washington, DC, p 63-78

Bolten AB, Balazs GH (1995) Biology of the early pelagic stage: the 'lost year'. In Bjorndal KA (ed) Biology and conservation of sea turtles, revised edn. Smithsonian Institution Press, Washington, DC, p 575-581

Bowen BW, Karl SA (2007) Population genetics and phylogeography of sea turtles. Mol Ecol 16:4886-4907

> Bowen BW, Avise JC, Richardson JI, Meylan AB, Margaritoulis D, Hopkins-Murphy SR (1993) Population structure of loggerhead turtles (Caretta caretta) in the northwestern Atlantic Ocean and Mediterranean Sea. Conserv Biol 7:834-844

Bowen BW, Bass AL, Chow SM, Bostrom M and others (2004) Natal homing in juvenile loggerhead turtles (Caretta caretta). Mol Ecol 13:3797-3808

Bowen BW, Bass AL, Soares L, Toonen RJ (2005) Conservation implications of complex population structure: lessons from the loggerhead turtle (Caretta caretta). Mol Ecol 14:2389-2402

Bradai MN, Karaa S, Jribi I, Elouaer A and others (2008) Rapport relatif au réseau national d'échouages des tortues marines et des cétacés: Bilan 2006-2007. INSTM, Tunis

> Broderick AC, Coyne MS, Fuller WJ, Glen F, Godley BJ (2007) Fidelity and overwintering of sea turtles. Proc R Soc Lond B Biol Sci 274:1533-1538

> Cardona L, Revelles M, Carreras C, San Félix M, Gazo M, Aguilar A (2005) Western Mediterranean immature loggerhead turtles: habitat use in spring and summer assessed through satellite tracking and aerial surveys. Mar Biol 147:583-591

> Carreras C, Pont S, Maffucci F, Pascual M and others (2006) Genetic structuring of immature loggerhead sea turtles (Caretta caretta) in the Mediterranean Sea reflects water circulation patterns. Mar Biol 149:1269-1279

> Carreras C, Pascual M, Cardona L, Aguilar A and others (2007) The genetic structure of the loggerhead sea turtle (Caretta caretta) in the Mediterranean as revealed by nuclear and mitochondrial DNA and its conservation implications. Conserv Genet 8:761-775

Carreras C, Pascual M, Cardona L, Marco A and others
(2011) Living together but remaining apart: Atlantic and Mediterranean loggerhead sea turtles (Caretta caretta) in shared feeding grounds. J Hered 102:666-677

Casale P, Margaritoulis D (2010) Sea turtles in the Mediterranean: distribution, threats and conservation priorities. IUCN/SSC Marine Turtle Specialist Group. IUCN, Gland

> Casale P, Freggi D, Basso R, Vallini C, Argano R (2007) A model of area fidelity, nomadism and distribution patterns of loggerhead sea turtles (Caretta caretta) in the Mediterranean Sea. Mar Biol 152:1039-1049

Casale P, Abbate G, Freggi D, Conte N, Oliverio M, Argano R (2008a) Foraging ecology of loggerhead sea turtles Caretta caretta in the central Mediterranean Sea: evidence for a relaxed life history model. Mar Ecol Prog Ser 372:265-276

Casale P, Freggi D, Gratton P, Argano R, Oliverio M (2008b) Mitochondrial DNA reveals regional and interregional importance of the central Mediterranean African shelf for loggerhead sea turtles (Caretta caretta). Sci Mar 72: 541-548

Chaieb O, El Ouaer A, Maffucci F, Bradai MN, Bentivegna F, Said K, Chatti N (2010) Genetic survey of loggerhead turtle Caretta caretta nesting population in Tunisia. Mar Biodiv Rec 3(e20):1-6

> Chenna R, Sugawara H, Koike T, Lopez R, Gibson TJ, Higgins DG, Thompson JD (2003) Multiple sequence alignment with the Clustal series of programs. Nucleic Acids Res 31:3497-3500

> Crouse DT, Crowder LB, Caswell H (1987) A stage-based population model for loggerhead sea turtles and implications for conservation. Ecology 68:1412-1423

> Davenport J, Clough W (1986) Swimming and diving in young loggerhead sea turtles (Caretta caretta L.). Copeia 1986:53-57

Echwikhi K, Jribi I, Bradai MN, Bouain A (2010) Gillnet fishery-loggerhead turtle interactions in the Gulf of Gabes, Tunisia. Herpetol J 20:25-30

> Eckert SA, Moore JE, Dunn DC, Van Buiten RS, Eckert KL, Halpin PN (2008) Modeling loggerhead turtle movement in the Mediterranean: importance of body size and oceanography. Ecol Appl 18:290-308

Ehrhart LM, Bagley DA, Redfoot WE (2003) Loggerhead turtles in the Atlantic Ocean: geographic distribution, abundance, and population status. In: Bolten AB, Witherington B (eds) Loggerhead sea turtles. Smithsonian Institution Press, Washington, DC, p 157-174

- Encalada SE, Bjorndal KA, Bolten AB, Zurita JC and others (1998) Population structure of loggerhead turtle (Caretta caretta) nesting colonies in the Atlantic and Mediterranean regions as inferred from mtDNA control region sequences. Mar Biol 130:567-575

Excoffier L, Laval G, Schneider S (2005) Arlequin ver. 3.0: an integrated software package for population genetics data analysis. Evol Bioinform Online 1:47-50

> Garofalo L, Mingozzi T, Mico A, Novelletto A (2009) Loggerhead turtle (Caretta caretta) matrilines in the Mediterranean: further evidence of genetic diversity and connectivity. Mar Biol 156:2085-2095

Gelman A, Rubin DB (1992) Inference from iterative simulation using multiple sequences. Stat Sci 7:457-511

> Giovannotti M, Franzellitti S, Cerioni P, Fabbri E and others (2010) Genetic characterization of loggerhead turtle (Caretta caretta) individuals stranded and caught as bycatch from the north-central Adriatic sea. AmphibReptilia 31:127-133 
Hall TA (1999) BioEdit: a user-friendly biological sequence alignment editor and analysis program for Windows 95/98/NT. Nucleic Acids Symp Ser 41:95-98

Hattour A (1999) Rapport final des activités de recherche sur les grands pélagiques des eaux tunisiennes. FAOCOPEMED/THONIDES.

Hays GC, Marsh R (1997) Estimating the age of juvenile loggerhead sea turtles in the North Atlantic. Can J Zool 75: 40-46

> Hays GC, Fossette S, Katselidis KA, Mariani P, Schofield G (2010) Ontogenetic development of migration: Lagrangian drift trajectories suggest a new paradigm for sea turtles. J R Soc Interface 7:1319-1327

Hillis DM, Mable BK, Larson A, Davis SK, Zimmer EA (1996) Nucleic acids IV: sequencing and cloning. In: Hillis DM, Moritz C, Mable BK (eds) Molecular systematics. Sinauer Associates, Sunderland, MA, p 321-381

> Hochscheid S, Bentivegna F, Bradai MN, Hays GC (2007) Overwintering behaviour in sea turtles: dormancy is optional. Mar Ecol Prog Ser 340:287-298

Jribi I, Bradai MN, Bouain A (2007) Impact of trawl fishery on marine turtles in the Gulf of Gabès, Tunisia. Herpetol J 17:110-114

Kass RE, Carlin BP, Gelman A, Neal RM (1998) Markov chain Monte Carlo in practice: a roundtable discussion. Am Stat 52:93-100

Laurent L, Casale P, Bradai MN, Godley BJ and others (1998) Molecular resolution of the marine turtle stock composition in fishery bycatch: a case study in the Mediterranean. Mol Ecol 7:1529-1542

Limpus CJ, Reed PC (1985) The green turtle, Chelonia mydas in Queensland: a preliminary description of the population structure in a coral reef feeding ground. In: Grigg G, Shine R, Ehmann H (eds) Biology of Australasian frogs and reptiles. Surrey Beatty \& Sons, Chipping Norton, p 47-52

Maffucci F, Kooistra WHCF, Bentivegna F (2006) Natal origin of loggerhead turtles, Caretta caretta, in the neritic habitat off the Italian coasts, Central Mediterranean. Biol Conserv 127:183-189

Margaritoulis D, Argano R, Baran I, Bentivegna F and others (2003) Loggerhead turtles in the Mediterranean Sea: present knowledge and conservation perspectives. In: Bolten $\mathrm{AB}$, Witherington $\mathrm{BE}$ (eds) Loggerhead sea turtles. Smithsonian Institution, Washington, DC, p 175-198

Mingozzi T, Masciari G, Paolillo G, Pisani B, Russo M, Massolo A (2007) Discovery of a regular nesting area of loggerhead turtle Caretta caretta in southern Italy: a new perspective for national conservation. Biodivers Conserv 16:3519-3541

> Monzon-Arguello C, Rico C, Naro-Maciel E, Varo-Cruz N, Lopez P, Marco A, Lopez-Jurado LF (2010) Population structure and conservation implications for the loggerhead sea turtle of the Cape Verde Islands. Conserv Genet 11:1871-1884

Naro-Maciel E, Becker JH, Lima EHSM, Marcovaldi MA, DeSalle R (2007) Testing dispersal hypotheses in foraging green sea turtles (Chelonia mydas) of Brazil. J Hered 98:29-39

Editorial responsibility: Paolo Casale, Rome, Italy
Narum SR (2006) Beyond Bonferroni: less conservative analyses for conservation genetics. Conserv Genet 7: 783-787

Nei M (1987) Molecular evolutionary genetics. Columbia University Press, New York, NY

Norman JA, Moritz C, Limpus CJ (1994) Mitochondrial DNA control region polymorphisms: genetic markers for ecological studies of marine turtles. Mol Ecol 3:363-373

> Onken R, Robinson AR, Lermusiaux PFJ, Haley PJ Jr, Anderson LA (2003) Data-driven simulations of synoptic circulation and transports in the Tunisia-Sardinia-Sicily region. J Geophys Res 108(C9):8123-8136

Owens D, Ruiz GW (1980) New methods of obtaining blood and cerebrospinal fluid from marine turtles. Herpetologica 36:17-20

Raymond M, Rousset F (1995) An exact test of population differentiation. Evolution 49:1280-1283

Reis EC, Soares LS, Vargas SM, Santos FR and others (2010) Genetic composition, population structure and phylogeography of the loggerhead sea turtle: colonization hypothesis for the Brazilian rookeries. Conserv Genet 11: 1467-1477

> Revelles M, Cardona L, Aguilar A, San Félix M, Fernández G (2007a) Habitat use by immature loggerhead sea turtles in the Algerian Basin (western Mediterranean): swimming behaviour, seasonality and dispersal pattern. Mar Biol 151:1501-1515

- Revelles M, Carreras C, Cardona L, Marco A and others (2007b) Evidence for an asymmetrical size exchange of loggerhead sea turtles between the Mediterranean and the Atlantic through the Straits of Gibraltar. J Exp Mar Biol Ecol 349:261-271

Revelles M, Caminas JA, Cardona L, Parga ML and others (2008) Tagging reveals limited exchange of immature loggerhead sea turtles (Caretta caretta) between regions in the western Mediterranean. Sci Mar 72:511-518

Schofield G, Hobson VJ, Fossette S, Lilley MKS, Katselidis KA, Hays GC (2010) Fidelity to foraging sites, consistency of migration routes and habitat modulation of home range by sea turtles. Divers Distrib 16:840-853

Spiegelhalter DJ, Tomas A, Best NG, Lunn D (2004) WinBUGS version 2.0 users manual. MRC Biostatistics Unit, Cambridge

SPSS (2010) PASW STATISTICS 18.0 Command Syntax Reference. SPSS, Chicago, IL

Tajima F (1983) Evolutionary relationship of DNA sequences in finite populations. Genetics 105:437-460

- Weir BS, Cockerham CC (1984) Estimating F-statistics for the analysis of population structure. Evolution 38:1358-1370

> Zbinden J, Aebischer A, Margaritoulis D, Arlettaz R (2007) Important areas at sea for adult loggerhead sea turtles in the Mediterranean Sea: Satellite tracking corroborates findings from potentially biased sources. Mar Biol 153: 899-907

> Zbinden JA, Bearhop S, Bradshaw P, Gill B, Margaritoulis D, Newton J, Godley BJ (2011) Migratory dichotomy and associated phenotypic variation in marine turtles revealed by satellite tracking and stable isotope analysis. Mar Ecol Prog Ser 421:291-302

Submitted: April 6, 2011; Accepted: February 23, 2012

Proofs received from author(s): June 28, 2012, 2012 